\title{
Harmonic Frequency Components Detection by a Guide Wave type Electromagnetic Acoustic Transducers [EMATs] *
}

\author{
Riichi MURAYAMA**, Kazumi AYAKA**, Kohei YAMAUCHI** \\ and Kazuki YOSHIDA** \\ **Fukuoka Institute of Technology, Fukuoka, Japan
}

\begin{abstract}
When an ultrasonic wave is injected into a crack, if the width of the crack opening is of almost the same order as the displacement of the ultrasonic wave, the crack may be closed or opened. As a result, the waveform of the received ultrasonic wave is different from that of the incident ultrasonic wave. Therefore, regarding the incident ultrasonic wave, its harmonic frequency components change as it penetrates the crack. However, the nonlinearity of a solid material is very low compared to that of a liquid medium, therefore, a liquid medium with a high nonlinear efficiency had to be used as a coupling medium. We then attempted to apply an electromagnetic acoustic transducer (EMAT), which does not require a coupling medium. In addition, we tried to develop an EMAT that could alternately drive a Lamb wave $\left(\mathrm{S}_{0}\right.$-mode, $\mathrm{A}_{0}$-mode $)$ and a Shear Horizontal $\left(\mathrm{SH}_{0}\right)$-plate wave to detect any nonlinearity in an ultrasonic wave. We actually tested the performance using fabricated fatigue specimens. As a result, we observed that the harmonic components increased when we used the specimen with a specific loading condition and a specific ultrasonic mode. This indicated that the harmonic component detection using the trial EMAT could also provide useful information on the damage to any structures or any materials
\end{abstract}

Key words: Nonlinearity, Ultrasonic Wave, EMAT, Lamb Wave, SH-Plate Wave, Fatigue Specimen

\section{Introduction}

The safety of a structure is important, and the early detection of any damage to a structure has been required in recent years. However, current nondestructive inspection techniques cannot meet this demand regardless of the technique being used. An ultrasonic inspection method, one of the nondestructive inspection techniques, also cannot satisfy this demand. In other words, we could not detect if a structure has a sufficient residual life. The technology using a nonlinear ultrasonic wave has been rapidly popularized as a high precision diagnosis technique of the human body in the medical field. However, the nonlinearity of a solid material is very low compared to that of a liquid medium. Therefore, the application to a solid material was in reality very difficult, although many researchers have been trying to detect minute defects using a nonlinear ultrasonic wave with many devices, because an ultrasonic transducer with a piezoelectric element requires a liquid medium with a high nonlinear efficiency as the coupling medium. Therefore, we have been studying a nonlinear ultrasonic technique using an electromagnetic transducer that is generally called an EMAT. In this paper, the experimental results of a trial sensing system with the EMAT using the modes of both a Lamb wave and a Shear Horizontal plate wave, that is generally called an SH-plate wave, are described.

\section{Principle of contact-type nonlinear ultrasonic wave ${ }^{(1)-(6)}$}

When the opening in a crack is of almost the same order as the displacement of the ultrasonic wave, the tensile stress induced by the ultrasonic wave will not be transmitted, while the resulting compression stress will be transmitted after the crack is closed in the 
compression phase. In this case, the ultrasonic wave repeats its passing or reflection movements, and as a result, the ultrasonic waveform is different from that of the injected ultrasonic wave. In contrast, if the crack penetrated width is greater than the displacement of the incident ultrasonic wave, the ultrasonic wave will not be transmitted through the crack. The magnitude of the ultrasonic wave displacement may be approximately estimated to be of a nanometer order. That is, the detection of a crack with a width of less than a nanometer order is considered possible by measuring the nonlinearity of the ultrasonic wave.

\section{Concepts of our research}

It has been postulated that the EMAT has poor ultrasonic wave generation efficiency and cannot detect a nonlinear ultrasonic wave of the plastic distortion type. However, even if its performance is limited, when the relationship between the width of a crack and the ultrasonic wave displacement was obtained under the contact-type nonlinear ultrasonic wave generation conditions, we considered that it was possible to detect a nonlinear ultrasonic wave using an EMAT. In addition, an EMAT, which does not use a coupling medium, may be able to perform a more exact evaluation compared to the contact-type ultrasonic transducer as shown in Fig.1.

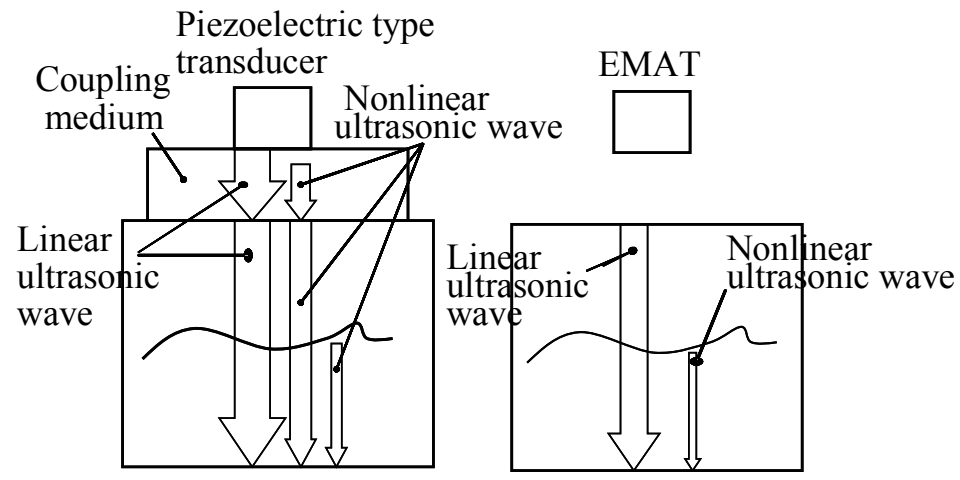

Fig.1 Comparison between a piezoelectric-type ultrasonic transducer and an electromagnetic acoustic transducer (EMAT)

\section{Ultrasonic wave mode}

Our objective was the detection of minute cracks in a thin steel sheet using a plate wave because an EMAT could generate it more easily compared to the other ultrasonic wave modes. Especially, we tested both a Lamb wave and an SH-plate wave such that their displacement direction intersects at a right angle. We actually designed it as the displacement directions of both ultrasonic wave modes were made to be same by intersecting the traveling direction at a right angle as shown in Fig.2.

O: An ultrasonic wave on compression stress side can pass the crack $X$ : An ultrasonic wave reflects at the crack

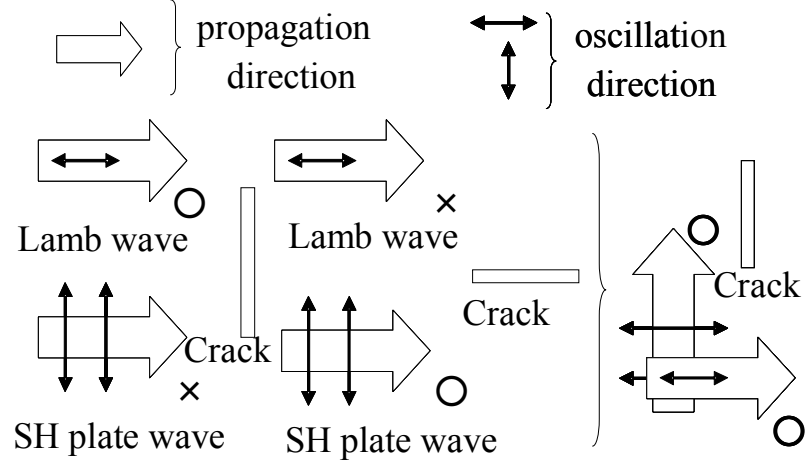

Fig.2 Relationship between the displacement direction and the crack direction 


\section{Basic structure of the trial EMAT for nonlinear ultrasonic wave detection}

Figure 3 shows the basic structure of the EMAT for a nonlinear ultrasonic wave detection (7)-(8). The EMAT consists of an electromagnet that produces a bias magnetic field, and two pairs of sensor coils. One of the pairs is to transmit and receive the Lamb wave and the other is to transmit and receive the SH-plate wave. Each sensor coil pair consists of three meandering sensor coils. One of the sensor coils is a transmitter that produces a dynamic magnetic field and the other two are receivers, one which can specifically detect the received signal having the fundamental drive frequency components and the other can specifically detect that having the double harmonic frequency components. We could then easily detect a nonlinear ultrasonic wave. These three sensor coils were placed parallel to the traveling direction of the plate wave between both magnetic poles. The driving force uses a high-frequency magnetostriction vibration generated in the direction of the compound's magnetic field by combining the dynamic magnetic field generated by a highfrequency electric current in the sensor coil and the static field due to the electromagnet. It could generate a Lamb wave when the electromagnet was set parallel to the sensor coil pair and could generate an SH plate wave when the electromagnet was set vertical to the sensor coil pair ${ }^{(9)-(10)}$. The distance between the electric fingers was determined using Ex. (1). Therefore, the distance between the electric fingers was $2.6 \mathrm{~mm}$ for the $\mathrm{S}_{0}$-mode Lamb wave, $0.5 \mathrm{~mm}$ for the $\mathrm{A}_{0}$-mode Lamb wave and $1.5 \mathrm{~mm}$ for the $\mathrm{SH}_{0}$ plate wave using a thin $0.5 \mathrm{~mm}$ thick steel sheet. The distance between the transmitter and receivers was typically $70 \mathrm{~mm}$. The transmitted signal to drive the transmitter is a four cycle burst-type pulse with a drive frequency of $1 \mathrm{MHz}$, and the maximum voltage was $1000 \mathrm{~V}$. The impedance of the transmitter at the $1 \mathrm{MHz}$ drive frequency was $4.3 \Omega$ on the test piece and the number of turns of the sensor coil was five. The amplifier is able to amplify the signal in the frequency range from $2 \mathrm{kHz}$ to $4 \mathrm{MHz}$ with an amplification magnitude of $40 \mathrm{~dB}$. The impedance of the receiver at the $2 \mathrm{MHz}$ drive frequency was $22.3 \Omega$ on the test piece and the number of turns of the sensor coil was ten. The applied voltage from the power supply to drive the electromagnet was $40 \mathrm{~V}$, and the resistance of the magnetic coil in the electromagnet was 4 $\Omega$. The number of turns of the magnetic coil was 700 .

$V(\mathrm{~km} / \mathrm{s})=F(\mathrm{MHz}) \times W(\mathrm{~mm}) \ldots$

$V$ denotes the phase velocity

$F$ denotes the drive frequency

$W$ denotes the wavelength

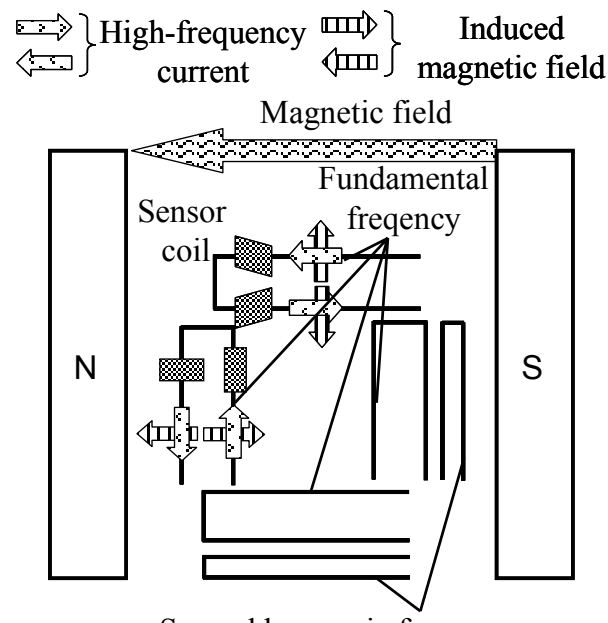

Second hurmonic frequency

Fig.3 Basic structure of EMAT for a nonlinear ultrasonic wave that alternately drives a Lamb wave and an SH-plate 


\section{Manufacturing conditions for test specimen}

We exceeded the fatigue examination using the tensile specimen in which a semicircle was produced on both ends in the center to produce a fatigue specimen with minute defects as shown in Fig.4 (a). The test specimen was cut out from the iron sheet which has a 99.5\% purity and $0.5 \mathrm{~mm}$ thickness. The load added for the test specimen was stress of one-side amplitude. Figure 4(b) shows the relation between time and the stress amplitude of the one-side amplitude. The cycle of the stress was $10 \mathrm{~Hz}$ and the stress ratio was assumed to be 0.1 so as not to become a compression test. When we assume $\sigma_{\max }$ as the maximum value of the stress during one cycle, we could calculate $\sigma_{\min }, \sigma_{\mathrm{a}}$ and $\sigma_{\text {mean }}$ using equations (2)-(4).

$$
\begin{aligned}
& \sigma_{\min }=\sigma_{\max } \times 0.1 \\
& \sigma_{a}=\frac{\sigma_{\max }+\sigma_{\min }}{2} \ldots(2) \\
& \sigma_{\text {mean }}=\frac{\sigma_{\max }-\sigma_{\min }}{2}+\sigma_{\min }
\end{aligned}
$$

The results of the fatigue test are shown in Fig.4 (c). We exceeded the fatigue examination under the condition of a periodic repetition of $10 \mathrm{~Hz}$. The number of repetition times when the tensile specimen was destroyed under different loads is indicated by the black lozenge mark. We then decided to manufacture the fatigue specimens using $\sigma$ max of $250 \mathrm{MPa}$ with $0,5,000,10,000,15,000,20,000,40,000$ and 80,000 repetition times which means that the rest lives were $100,95,85,75,50$ and $5 \%$, respectively. We finally manufactured four sheets, each under the same conditions. The surface of one sheet under the different load conditions was observed using an electron microscope. We confirmed that the cracks grew in all the specimens for a repetition number greater than 20000 times, and cracks having $\mu \mathrm{m}$ gaps were generated in all the specimens. We did not observe a crack under the load condition of 5000 times.

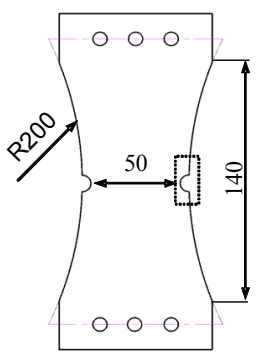

(a)Outline of the fatigue specimen

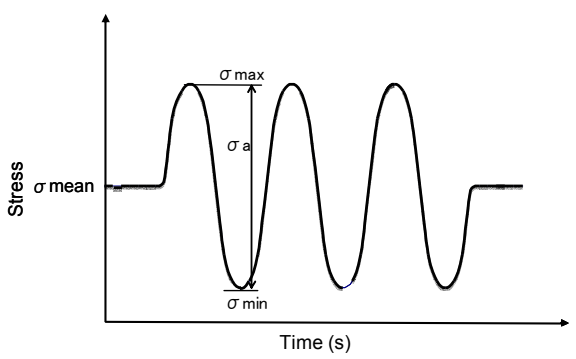

(b) Stress pattern of the fatigue test

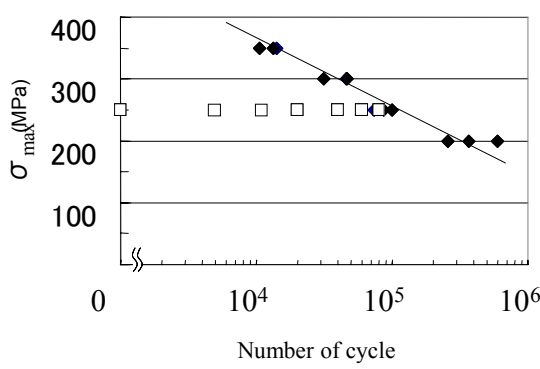

(c) Condition of the fatigue test

Fig.4 Fatigue specimen 


\section{Experimental system}

The system consists of the EMAT that generates a Lamb wave and an SH -plate wave, the one-dimensional scanner that can move the EMAT sensor with a maximum moving range of $150 \mathrm{~mm}$, the driving system that can apply a high power and a high-frequency electrical signal to the EMAT sensor, the receiving system that can amplifies by $40 \mathrm{~dB}$ the received electrical signal that the EMAT receiver could detect, the A/D converter with a $100 \mathrm{MHz}$ sampling frequency and CPU as shown in Fig.5.

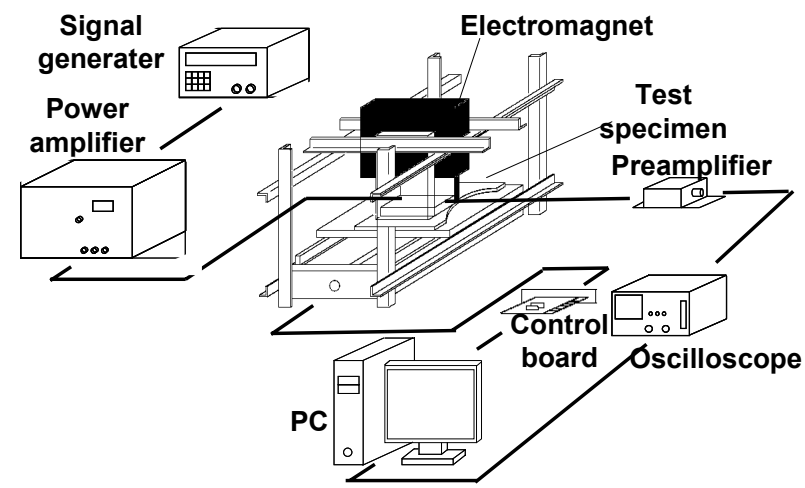

Figure 5 Experimental system

\section{Experimental results}

\subsection{Fundamental performance}

Figure 6 is the power spectrum of the received signal waveform by the received EMAT sensors for the fundamental frequency and double harmonic frequency. It was confirmed that the harmonic frequency components can be hardly observed when we used the receiver for the basic frequency, although we could observe the harmonic frequency components when we used the receiver for the harmonic frequency. We noted that the ratio of the intensity of the harmonic frequency (A2) and fundamental frequency (A1) to be a nonlinear parameter.

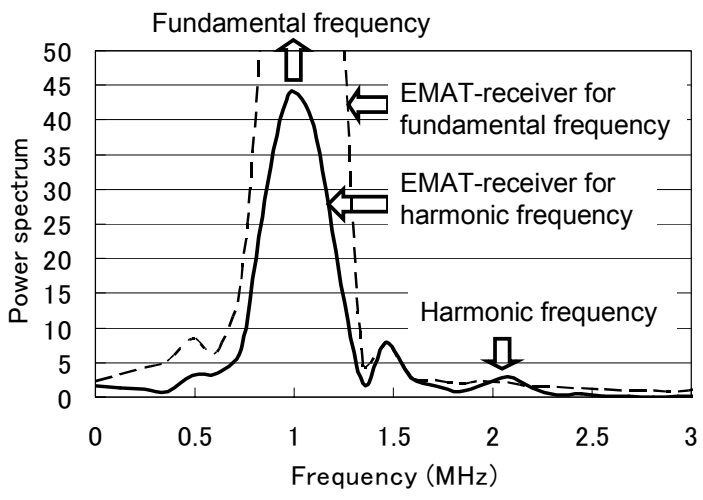

Fig.6 Example of the Fourier analysis results of the receiving signal waveform using the receivers for fundamental frequency and for harmonic frequency

Figure 7 shows in the change in the signal amplitude and the nonlinear ultrasonic index (A2/A1) due to the load condition. At first, we could confirm that the received signal amplitude was almost stable, i.e., the change was less than $1 \%$, although the received EMAT sensors were used or the load condition changed. Next, we could only very slightly detect the change in the nonlinear ultrasonic index (A2/A1) using the received EMAT sensor for the fundamental frequency; they were less than $5 \%$. On the other hand, we could observe the increase in the A2/A1 by about twice when we used the received EMAT sensor for the harmonic frequency. We confirmed the utility of the trial EMAT which consisted of 
the transmitter with a fundamental drive frequency and the receiver with a double harmonic receiving frequency.

$\mathrm{O}$ : Signal amplitude using the EMAT receiver for fundamental drive frequency $\triangle$ : Signal amplitude using the EMAT receiver for double harmonic frequency

: Nonlinear ultrasonic index using the EMAT receiver for fundamental drive frequency

$\boldsymbol{\Delta}$ : Nonlinear ultrasonic index using the EMAT receiver for double harmonic frequency

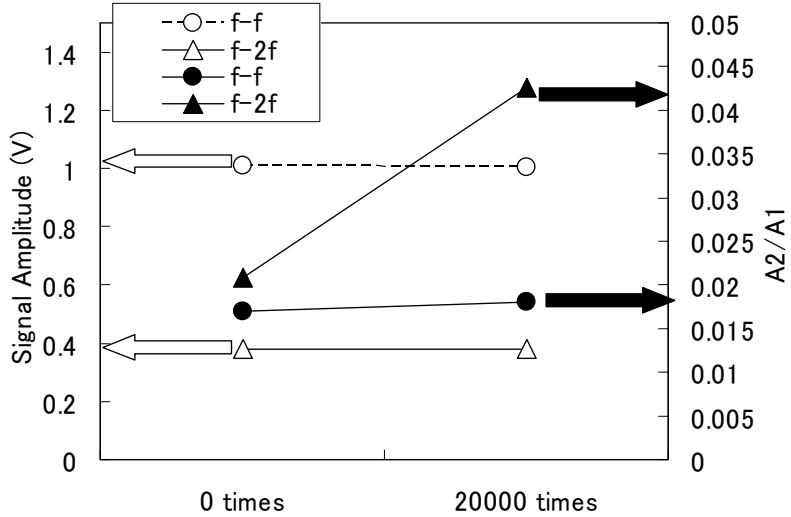

Fig.7 Relationship between the signal amplitude and load condition of the test specimen using the receiver for fundamental frequency and for harmonic frequency, relationship between the nonlinear ultrasonic (A2/A1) and the load condition of the test specimen using the receiver for fundamental frequency and for harmonic frequency

\subsection{Evaluation results using the fatigue specimen}

A comparative evaluation was done using the load-less test piece and the fatigue specimen with the EMAT for an $\mathrm{S}_{0}$ mode-lamb wave, an $\mathrm{A}_{0}$ mode-lamb wave and an $\mathrm{SH}_{0}$-plate wave. Fig.8 shows the measured nonlinear ultrasonic index (A2/A1) value when we changed the load condition and the measuring position using an $\mathrm{S}_{0}$-mode Lamb wave. It was confirmed that the measured nonlinear ultrasonic index (A2/A1) value had doubled between the fatigue specimen and the load-less test piece. We also confirmed that the difference in the measured nonlinear ultrasonic index (A2/A1) value using the load-less test piece and the fatigue specimen had decreased as the measurement point became farer from the center point of the test piece. This indicated that these measurement results corresponded to the damage level becoming farer from the center part of the test piece, as expected from the shape of the fatigue specimen. However, we could not observe any difference in the measured nonlinear ultrasonic index (A2/A1) value between the fatigue specimens with the different load conditions which were 0 time, 20,000 times, 40,000time and 80,000 times.

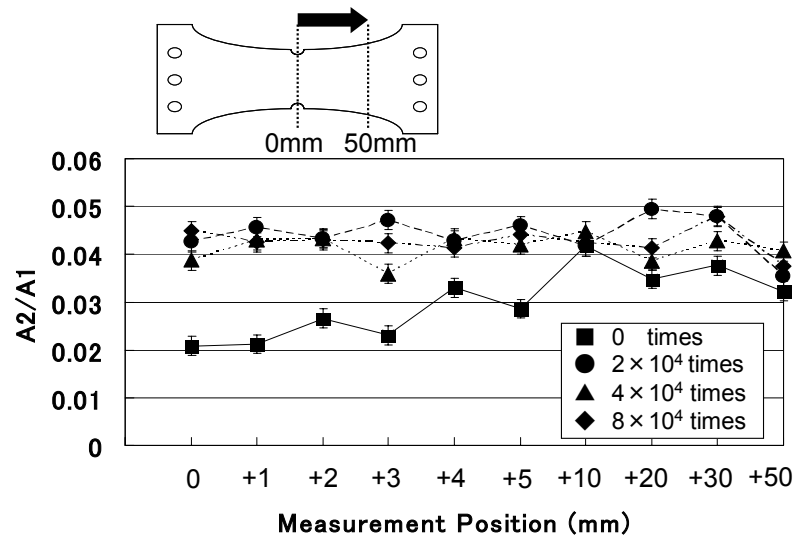

Fig.8 Relationship between the A2/A1 and the load condition, the measuring position by an $\mathrm{S}_{0}$-mode Lamb wave 
Figure 9 shows the measured nonlinear ultrasonic index (A2/A1) value when we changed the load condition and the measuring position using the $\mathrm{A}_{0}$-mode Lamb wave. It was confirmed that the measured nonlinear ultrasonic index (A2/A1) value did not change between the fatigue specimen and the load-less test piece. We also confirmed that the difference in the nonlinear ultrasonic index (A2/A1) value measured using the load-less test piece and the fatigue specimen had not changed as the measurement point became farer from the center point of the test piece. This indicated that we could not detect any damage in the test specimen using the nonlinear ultrasonic EMAT with the $\mathrm{A}_{0}$-mode Lamb wave.

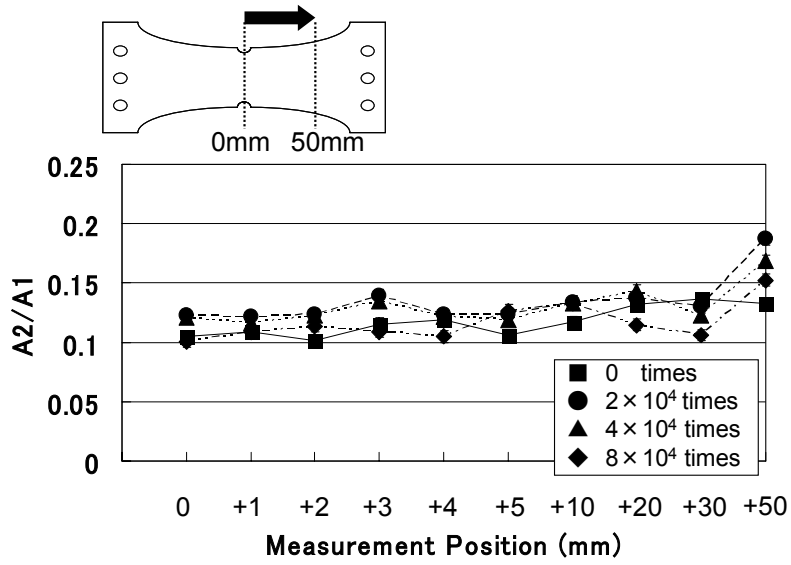

Fig.9 Relationship between the A2/A1 and the load condition, the measuring position by an $\mathrm{A}_{0}$-mode Lamb wave

Figure 10 shows the measured nonlinear ultrasonic index (A2/A1) value when we changed the load condition and the measuring position using the $\mathrm{SH}_{0}$-mode plate wave. It was confirmed that the A2/A1 had decreased approximately from 4 to 6 times between the fatigue specimen and the load-less test piece. We could not explain this phenomenon based on the nonlinear ultrasonic mutual interaction theory.

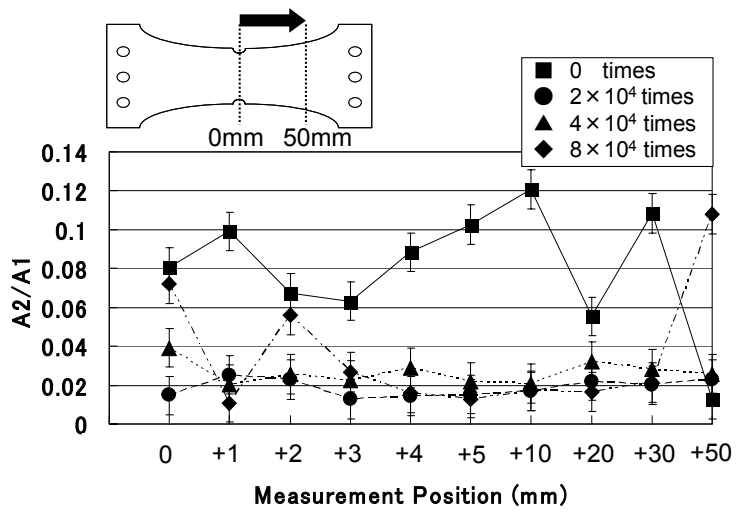

Fig.10 Relationship between the A2/A1 and the load condition, the measuring position by an $\mathrm{SH}_{0}$-mode Lamb wave

We evaluated the difference between the measured nonlinear ultrasonic index (A2/A1) value when the EMAT sensor is moving compared to the measured nonlinear ultrasonic index (A2/A1) value in the static state. Figure 11 shows these results. We confirmed that the difference in the measured nonlinear ultrasonic index (A2/A1) values had almost the same tendency when the EMAT sensor was moving or stopped, even if we used any ultrasonic mode. These results indicated that we could evaluate the damage degree of the materials or the structure using the nonlinear ultrasonic index (A2/A1) by the EMAT sensor for a nonlinear ultrasonic index parameter (A2/A1). 


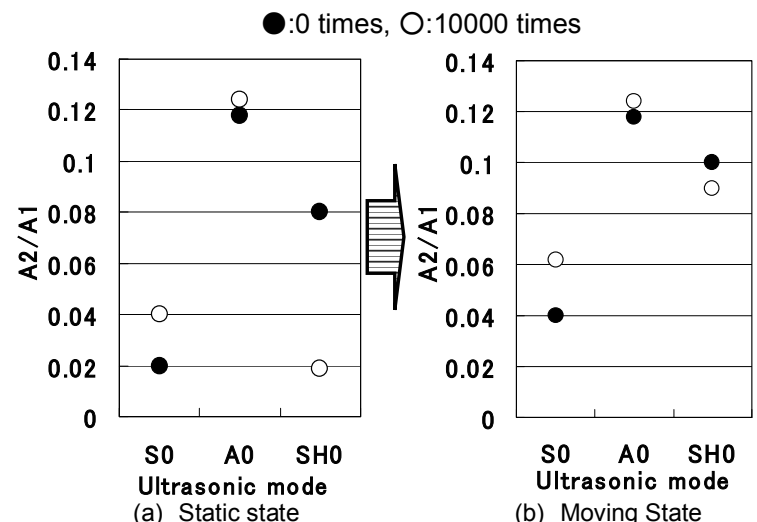

Fig.11 Difference in the measured A2/A1 value between the load-less test piece and the fatigue specimen when the EMAT sensor is moving or not moving

\section{Conclusions}

We tested the relationship between the damage degree of the test specimen and the nonlinear ultrasonic index (A2/A1) measured using the EMAT. We then confirmed that the nonlinear ultrasonic evaluation index (A2/A1) using the $\mathrm{S}_{0}$-mode Lamb wave had increased by twice between the fatigue specimen and the load-less test piece. We also confirmed that the difference in the nonlinear ultrasonic index (A2/A1) measured using the load-less test piece and the fatigue specimen had decreased as the measurement point became farer from the center point of the test piece. This indicated that it may be possible to detect the damage in the test specimen. However, we could not observe any difference in the nonlinear ultrasonic index (A2/A1) between the fatigue specimens with the different load conditions using an $\mathrm{A}_{0}$-mode ultrasonic mode. We also observed that the relationship between the degree of the test specimen and the nonlinear ultrasonic index (A2/A1) measured using the nonlinear ultrasonic EMAT that could drive an $\mathrm{SH}_{0}$-plate wave had the reverse tendency when compared to the nonlinear ultrasonic interaction theory.

We could indicate the utility of the trial EMAT for a nonlinear ultrasonic wave. However, it was confirmed there were several problems to still solve before use in nondestructive inspections. We are challenged to improve the sensor system and to clarify the reasons for these phenomena.

\section{Acknowledgement}

This work was partially supported by The Japan Science and Technology Agency (JST) through a Research for Promoting Technological Seeds, 12-042, 2006 and Grants-in-Aid for Scientific Research (c), 19560104, 2007.

\section{References}

1. Alippi, A., Bettucci, A., Germano, M. and Passeri, D., Ultrasonics, Vol.44 (2006), pp.1313-1318.

2. Deng, M., Ultrasonics, Vol.44 (2006), pp.1157-1162.

3. Kim, J.-Y., Qu, j., Jacobs, L. J., Littles, J. W. and Savage, M. F., Journal of Nondestructive Evaluation, Vol.25, No.1 (2006), pp.28-36.

4. Kawashima, K., Omote, R., Ito, T., Fujita, H. and Shima, T., Ultrasonics, Vol.40 (2001), pp.611-615.

5. Okada, J., Ito, T., Fujita, H., Kawashima, K. and shimura, N., Japanese Journal of Applied Physics, Vol.40 (2001), pp.35793582.

6. Ogi, H., Hirao M. and Aoki, S., Journal of Applied Physics, Vol.90 (2001), pp.438-442.

7. Thompson, R. B., IEEE Trans. on Sonics and Ultrasonics, SU-25 (1978), pp.7-15.

8. Murayama, R. and Mizutani, K., Japanese Journal of Applied Physics, Vol.41 (2002), pp.3534-3538.

9. Murayama, R., Hoshihara, H. and Fukushige, T., Japanese Journal of Applied Physics, Vol.42 (2003), pp.3180-3183.

10. Murayama, R., Makiyama, S., Kodama, M. and Taniguchi. Y., Ultrasonics, Vol.42 (2004), pp.825-829. 